\title{
Coexistence of structured populations with size-based prey selection
}

\author{
Hartvig, Martin; Andersen, Ken Haste
}

Published in:

Theoretical Population Biology

Link to article, DOI:

10.1016/j.tpb.2013.07.003

Publication date:

2013

Link back to DTU Orbit

Citation (APA):

Hartvig, M., \& Andersen, K. H. (2013). Coexistence of structured populations with size-based prey selection. Theoretical Population Biology, 89, 24-33. https://doi.org/10.1016/j.tpb.2013.07.003

\section{General rights}

Copyright and moral rights for the publications made accessible in the public portal are retained by the authors and/or other copyright owners and it is a condition of accessing publications that users recognise and abide by the legal requirements associated with these rights.

- Users may download and print one copy of any publication from the public portal for the purpose of private study or research.

- You may not further distribute the material or use it for any profit-making activity or commercial gain

- You may freely distribute the URL identifying the publication in the public portal

If you believe that this document breaches copyright please contact us providing details, and we will remove access to the work immediately and investigate your claim. 
Theoretical Population Biology, Volume 89, November 2013, Pages 24-33 http://dx.doi.org/10.1016/j.tpb.2013.07.003 @ 2013 Elsevier Inc.

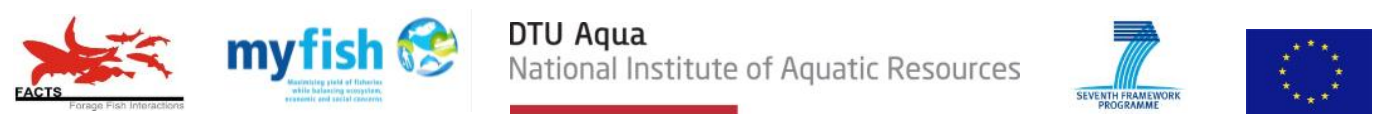

$\underline{\text { www.facts-project.eu/ } \quad \text { www.aqua.dtu.dk } \quad \text { http://cordis.europa.eu/fp7/ }}$

\title{
Coexistence of structured populations with size-based prey selection
}

\author{
Martin Hartvig $\underline{\mathrm{a}}, \underline{\mathrm{b}}, \underline{\mathrm{c}}, *$, Ken Haste Andersen ${ }^{\mathrm{b}}, \underline{\mathrm{d}}$ \\ ${ }^{a}$ Center for Macroecology, Evolution and Climate, University of Copenhagen, Universitetsparken 15, DK- \\ 2100 Copenhagen, Denmark \\ ${ }^{\mathrm{b}}$ National Institute of Aquatic Resources, Technical University of Denmark, Charlottenlund Slot, \\ Jægersborg Allé 1, DK-2920 Charlottenlund, Denmark \\ ${ }^{c}$ Department of Biology, Lund University, Ecology Building, SE-223 62 Lund, Sweden \\ ${ }^{d}$ Centre for Ocean Life, National Institute of Aquatic Resources, Denmark
}

\begin{abstract}
Species with a large adult-offspring size ratio and a preferred predator-prey mass ratio undergo ontogenetic trophic niche shift(s) throughout life. Trophic interactions between such species vary throughout life, resulting in different species-level interaction motifs depending on the maximum adult sizes and population size distributions. We explore the assembly and potential for coexistence of small communities where all species experience ontogenetic trophic niche shifts. The life-history of each species is described by a physiologically structured model and species identity is characterised by the trait: size at maturation. We show that a single species can exist in two different states: a 'resource driven state' and a 'cannibalistic state' with a large scope for emergent Allee effects and bistable states. Two species can coexist in two different configurations: in a 'competitive coexistence' state when the ratio between sizes at maturation of the two species is less than a predator-prey mass ratio and the resource level is low to intermediate, or in a 'trophic ladder' state if the ratio of sizes at maturation is larger than the predator-prey mass ratio at all resource levels. While there is a large scope for coexistence of two species, the scope for coexistence of three species is limited and we conclude that further trait differentiation is required for coexistence of more species-rich size-structured communities.
\end{abstract}

Keywords: Species interactions; Intraguild predation; Cannibalism; Community assembly; Life-history omnivory; Trait-based model

*Corresponding author: $\underline{\text { mh@hvig.dk }}$

Article first published online: 6 Aug 2013

Please note that this is an author-produced PostPrint of the final peer-review corrected article accepted for publication. The definitive publisher-authenticated version can be accesses here: http://dx.doi.org/10.1016/i.tpb.2013.07.003 @ 2013 Elsevier Inc. 


\title{
Coexistence of structured populations with size-based prey selection
}

\author{
Martin Hartviga,b,c,1, Ken Haste Andersen ${ }^{\mathrm{b}, \mathrm{d}}$ \\ ${ }^{a}$ Center for Macroecology, Evolution and Climate, University of Copenhagen, Universitetsparken 15, DK-2100 \\ Copenhagen, Denmark \\ ${ }^{b}$ National Institute of Aquatic Resources, Technical University of Denmark, Charlottenlund Slot, Jagersborg Allé \\ 1, DK-2920 Charlottenlund, Denmark \\ ${ }^{c}$ Department of Biology, Lund University, Ecology Building, SE-223 62 Lund, Sweden \\ ${ }^{d}$ Centre for Ocean Life, National Institute of Aquatic Resources, Denmark
}

\begin{abstract}
Species with a large adult-offspring size ratio and a preferred predator-prey mass ratio undergo ontogenetic trophic niche shift(s) throughout life. Trophic interactions between such species vary throughout life, resulting in different species-level interaction motifs depending on the maximum adult sizes and population size distributions. We explore the assembly and potential for coexistence of small communities where all species experience ontogenetic trophic niche shifts. The life-history of each species is described by a physiologically structured model and species identity is characterized by the trait: size at maturation. We show that a single species can exist in two different states: a 'resource driven state' and a 'cannibalistic state' with a large scope for emergent Allee effects and bistable states. Two species can coexist in two different configurations: in a 'competitive coexistence' state when the ratio between sizes at maturation of the two species is less than a predator-prey mass ratio and the resource level is low to intermediate, or in a 'trophic ladder' state if the ratio of sizes at maturation is larger than the predator-prey mass ratio at all resource levels. While there is a large scope for coexistence of two species, the scope for coexistence of three species is limited and we conclude that further trait differentiation is required for coexistence of more species-rich size-structured communities.
\end{abstract}

Keywords: species interactions, intraguild predation, cannibalism, community assembly, life-history omnivory, trait-based model

\section{Introduction}

The ecological role of fish in aquatic communities is shaped by two characteristics: 1) individuals have a size-based preference for prey 10-1000 times smaller than themselves in terms of weight (Brose et al., 2006; Barnes et al., 2010), and 2) they have a life-history where they produce many small offspring, around $1 \mathrm{mg}$, which is several orders of magnitude smaller than their adult size ranging from $1 \mathrm{~g}$ to $100 \mathrm{~kg}$ (Duarte and Alcaraz, 1989; Winemiller and Rose, 1993; Andersen et al., 2008). Consequently this means that individuals feed on prey from successively higher trophic levels throughout ontogeny: as larvae individuals compete for shared resources of zooplankton nauplii, and as they grow they shift to adult zooplankton and later to piscivory and feeding on larger invertebrates. Individuals therefore undergo ontogenetic niche shifts (Werner and Gilliam, 1984) and specifically shifts in the trophic niche (also known as life-history omnivory; Pimm and Rice, 1987). Due to ontogenetic trophic niche shifts two individuals may interact either competitively, if they are of a similar size, or as predator and prey if the size of one individual falls into the preferred prey size range of the other. The ecological role is therefore

${ }^{*}$ Corresponding author; mh@hvig.dk (M. Hartvig) 
determined by the size of an individual rather than by its species identity. This complication makes it difficult to characterize the interaction between two fish populations as "competitive" or "predator-prey" since the species-level interaction type is likely to be a complex mixture of both that depends on the size structure of the two populations (Fig. 1).

The classic way of describing species-level interactions is through unstructured models based on coupled ordinary differential equations (e.g. MacArthur and Levins, 1967; Oksanen et al., 1981; Holt and Polis, 1997). In unstructured models energy is transferred between species only through predator-prey interactions. In populations with ontogenetic trophic niche shifts energy is also transferred through somatic growth in body size. Explicit modelling of ontogenetic trophic niche shifts therefore requires the use of physiologically structured modelling approaches (Metz and Diekmann, 1986; de Roos and Persson, 2001, 2013). The key characteristic of physiologically structured models is that they resolve food-dependent growth and reproduction, where growth can either be between stages (de Roos et al., 2008a), in a cohort model with several state variables (de Roos and Persson, 2001), or with a continuous size structure (Claessen and de Roos, 2003; Hartvig et al., 2011). The dependence of growth and reproduction on food encounter is what distinguishes physiologically structured models from the traditional age-based models used in fisheries science (Beverton and Holt, 1957). Analysis of physiologically structured models have e.g. demonstrated coexistence of several unstructured predator populations on a single structured prey population through the mechanism of emergent facilitation (de Roos et al., 2008b). It seems, however, that inclusion of ontogenetic trophic niche shifts decreases the possibility of coexistence of two size-structured species (van de Wolfshaar et al., 2006).

We explore the potential for coexistence of species experiencing ontogenetic trophic niche shifts. To simplify matters we assume that selection of food is determined solely by the differences in body size of the interacting individuals - meaning that we disregard any dietary selection based on species identity. We refer to such populations as "purely size-structured". We apply a continuously size-structured approach where individual physiological processes, including predator-prey encounter, are parameterized by relations with the size of individuals. In this manner each individual is characterised by body size, while each species is characterized by a single trait: the size at maturation (Hartvig et al., 2011). The formulation of purely size-structured populations through a single trait allows for a general analysis of coexistence as variation of a larger set of species-specific parameters are avoided. The analysis is divided into three steps: we first characterize a resource-consumer system where one structured species feeds on a size-structured resource and potentially on itself through cannibalism. In this system we identify the resource-driven and cannibalistic population states described earlier (Claessen and de Roos, 2003) including an emergent Allee effect (de Roos et al., 2003) and bistable states (Claessen and de Roos, 2003; van Kooten et al., 2005; Guill, 2009). Next we show how two species may coexist. Depending on the relative sizes at maturation the two species can be considered part of different species-level interaction motifs. If the sizes at maturation are similar, the two species will mainly interact as competitors for the resource. If size at maturation of one species is significantly larger than the other species the interaction is a combination of competitive coexistence and predator-prey interactions (including cannibalism). Finally we explore the potential for coexistence of three or more species and show how the scope for coexistence decreases rapidly when more species are introduced into the system.

\section{Model}

We employ a recent size- and trait-based model framework to describe the population dynamics of purely size-structured interacting populations (Hartvig et al., 2011). The model differs from the Hartvig et al. (2011) model only by assuming that interaction strengths between species is solely due to size-dependent predation (i.e. setting the species-specific couplings strengths to 
unity). The model formalizes the sketch in Fig. 1 by explicitly resolving the entire individual life-history from offspring size, through maturation and ultimately death by resolving the bioenergetics of individuals and the size distribution of each population. Individuals of the same size across species compete for food on an equal basis, but may have different growth potential depending on maturity as less energy is available for growth when reproductive investments are made. Small individuals feed exclusively on resources. As they grow in size they consume progressively larger prey and eventually they start feeding on the other species and cannibalise on smaller conspecifics. This is a generalisation of the resource competition and predator-prey interactions occurring between two species based on life-history theory and explicit individual-level ecological processes.

The model is a physiologically structured population model (Metz and Diekmann, 1986; de Roos and Persson, 2001, 2013) where the parameterization of the processes is based on three fundamental assumptions: 1) individual processes and interactions are characterized by individual body mass $m ; 2$ ) individuals ignore prey species identity and consider only relative prey size when selecting prey through an implementation of "big individuals eat smaller individuals" (Warren and Lawton, 1987; Cohen et al., 1993; Brose et al., 2006; Barnes et al., 2010) (which implies that all species have the potential of being cannibalistic); and 3) species are characterized by their size at maturation $m^{*}$. The remaining assumptions are standard descriptions of encounter, consumption, and a bioenergetic budget of growth and reproduction. The solution of the model is the continuous population structure of each species $i$ represented as a size-spectrum $N_{i}(m, t)$ at time $t . N_{i}(m, t)$ is the number density of individuals as a function of body mass $m$ and $N_{i}(m, t) d m$ is the number of individuals in the size range $[m, m+d m]$. The dynamics of each species' size-spectrum is governed by a conservation equation (M'Kendrick, 1926; von Foerster, 1959) stating that changes in number density at size $m$ is determined by somatic growth $g_{i}(m)$ and mortality $\mu_{i}(m)$ :

$$
\frac{\partial N_{i}}{\partial t}+\frac{\partial}{\partial m}\left(g_{i} N_{i}\right)=-\mu_{i} N_{i} .
$$

The model equations and parameters are given in Tables 1 and 2. The biotic environment an individual experiences provides food for growth and reproduction as well as enemies which may consume the individual. This environment is represented by the community size-spectrum $N_{c}(m)$, which is the sum of the species spectra and a resource spectrum (M1). The resource spectrum provides a continuous size range of resource items up to mesoplankton $\left(m_{\text {cut }}=0.5 \mathrm{~g}\right.$, Table 2). The dynamics of each size class is modelled with semi-chemostatic growth (M2). Carrying capacity of the resource spectrum is a power-law function $\mathrm{\kappa m}^{-\lambda}$ (Hartvig et al., 2011), and the regeneration rate is $r_{0} m^{-1 / 4}$ (Fenchel, 1974; Savage et al., 2004). Growth $g_{i}(m)$ of an individual is determined by the amount of food encountered and by the allocation of assimilated food between growth and reproduction. Individuals search the biotic environment for food with a volumetric search rate which increases with size (M3). Preference for prey size is described by a bell-shaped function with a preferred predator-prey ratio $\beta$ and a width $\sigma$ (M4) (Ursin, 1973, 1974). From these processes the encountered food of suitably sized prey is calculated (M5). Consumption of encountered food is limited by a type II functional response $f(\mathrm{~m}) \mathrm{hm} \mathrm{m}^{n}$ where $h m^{n}$ is the maximum consumption rate, and $f(m)$ the feeding level (M6) describing the degree of satiation of the functional response as a number between 0 and 1. Food is assimilated with efficiency $\alpha$ and metabolic costs $\mathrm{km}^{p}$ are subtracted. The remaining energy (M7) is available for growth and reproduction. The fraction of available energy routed to reproduction is controlled by $\psi\left(m, m^{*}\right)$, which represents a smooth transition to maturation around $m=m^{*}$ and, following that, and increasing investment to reproduction with size ensuring that the allocation to reproduction is proportional to individual mass (M8). The remaining energy is used for somatic growth (M9). The theoretical maximum asymptotic size an individual can obtain is $M=m^{*} / \eta$ where all available energy is routed into reproduction $(\psi=1)$. Growth may however stop at a 
smaller size if the metabolic costs exceed the assimilated food intake (stunted growth).

Energy routed to reproduction is converted with efficiency $\epsilon$ to offspring of size $m_{0}$. Mathematically this is described as a boundary condition $g_{i}\left(m_{0}\right) N_{i}\left(m_{0}\right)=R_{i}$ in (1). The flux of offspring $R_{i}$ is calculated from the energy routed to reproduction and multiplied with $1 / 2$ since only half the population is assumed to be females (M10).

Mortality $\mu_{i}(m)$ is partitioned into contributions from predation by larger individuals, starvation and a constant background mortality. The predation mortality can be derived by considering the ratio between the consumption of a predator of size $m$ to the total amount of food available to the predator:

$$
\mu_{p}\left(m_{p}\right)=\int \frac{s\left(m_{p} / m\right) f(m) h m^{n} N_{c}(m)}{\int s(\tilde{m} / m) N_{c}(\tilde{m}) \tilde{m} d \tilde{m}} d m .
$$

By using the definition of the functional response (M6) one of the integrals may be eliminated resulting in the simpler expression (M11).

Starvation mortality occurs when consumption is insufficient to cover metabolic costs, and is assumed proportional to the energy deficit and inversely proportional to reserve size (M12). Reserve size is assumed to be a fixed fraction $\xi$ of the body size $m$. Background mortality is needed to ensure that the largest individuals experience mortality. This mortality represents death by other natural causes than predation and starvation as e.g. illness or senescence. We assume that investments in defence and repair are balanced such that the background mortality is inversely proportional to generation time, which has an allometric scaling of $\left(m^{*}\right)^{1-n}$ (Peters, 1983) (M13).

Since all processes are scaled with $m$ or $m^{*}$ the parameters are species- and size-independent (Table 2; see Hartvig et al. (2011), App. E for parameter estimation). All differences between species are therefore characterised solely by the trait $m^{*}$. The physiological impact of the resources may be characterised by the initial feeding level $f_{0}$, which is the feeding level (functional response satiation) that small individuals experience when feeding on a resource spectrum at carrying capacity. The relevant range of $f_{0}$ is from the critical feeding level, $f_{c}=k /(\alpha h) m^{p-n}=k /(\alpha h) \approx 0.2$ (Hartvig et al., 2011), where the resource can just cover the metabolic requirements, to $f_{0} \rightarrow 1$ where individuals are satiated. $f_{0}$ is used to eliminate the search rate constant $\gamma$ from the set of parameters by expressing it in terms of $f_{0}$ (Table 2, and Hartvig et al. 2011, App. D). The size scaling exponent of the carrying capacity is set to the equilibrium solution scaling of a complete community $\lambda=2-n+q=2.05$, where $q$ is the exponent of the volumetric search rate (Andersen and Beyer, 2006; Hartvig et al., 2011).

\section{Methods}

The model (1) consists of coupled partial integro-differential equations and is solved numerically using a first order semi-implicit upwind finite-difference scheme (Press et al., 1992; Hartvig et al., 2011). The size-spectrum of all species is discretized on a mass grid $m_{0} \leq m \leq 85 \mathrm{~kg}$ with 200 logarithmically even sized mass $(m)$ groups, and the time step used for integration is 0.02 years. The dynamics of the resource spectrum may be obtained analytically at each time step to save computational time (Hartvig et al., 2011, App. G). The grid for the resources ends at $m_{c u t}$, and the lower limit is 3 decades lower than $m_{0}$ to avoid any boundary effect for small resources. Identical logarithmic grid spacing is used for the resources and species to ease computations in the overlap $\left[m_{0} ; m_{c u t}\right]$. It was checked that the results do not depend on the chosen discretisation.

The ecologically relevant phase space to examine coexistence in is spanned by resource level $f_{0}$ and the trait $m^{*}$. Depending on the specific configuration the system may either be in an 
equilibrium or have oscillating solutions. Since our main concern is whether species coexist or not, we disregard the dynamical aspects of the solution and average all spectra over time after the initial transient has passed. The potential for coexistence of the resource with one or two species is examined using simulations (Appendix A). Coexistence of more than two species is examined through sequential community assembly where an additional species is introduced in small numbers and simulated forward in time (Post and Pimm, 1983; Drake, 1990; Law, 1999).

\section{Results}

The analysis of a system with just one species demonstrates (Fig. 2): 1) There is an upper boundary to the size at maturation $m^{*}$ of a single species that can invade from small numbers (thick solid line). This maximum size of $m^{*}$ is determined by the resource level described by the initial feeding level $f_{0}$; higher resource levels leads to larger maximum size. 2) Beyond the invasion boundary species may persist but they cannot invade; these states can be reached by starting simulations from a high initial abundance level and appropriate population structure. This mean that an Allee effect exists in this region as the individual fitness at low density is so reduced that it leads the population to a sudden extinctions. 3) This Allee effect extends into the area where a species can invade leading to the appearance of bistability (hatching patterns overlap). The two types of states are termed 'resource driven' and 'cannibalistic' states (explained below). 4) The area with bistability is most prominent at intermediate resource levels. 5) At high resource levels it is possible to invade into the cannibalistic state. 6) A continuous transition between the two states exists at high resource levels and intermediate sizes at maturation (Fig. 2, top left quadrant where the hatching type switches).

The two states can be characterized through differences in their size-spectrum (Fig. 3). The specific shape of a size-spectrum is determined by the rates of growth and mortality. The two types of states in the bistable regime (Fig. 2) are characterised by different growth trajectories due to differences in feeding levels (Fig. 3). In the state which can be reached by invasion in small numbers (black line) individuals feed mainly on the resource. This leads to a depletion of the resource and consequently a drop in the feeding level just above the size of maturation, which causes a "growth bottleneck" where the growth slows down. This bottleneck results in a stunted size-spectrum where large adults are missing from the population. This state is referred to as the resource-driven state. The other state (grey line) has a higher abundance of small and medium sized individuals that allow small adults to avoid the energetic bottleneck by cannibalising on conspecifics. The higher abundance of juveniles causes a stronger depletion of smaller resources, which results in a bottleneck in the feeding level for small juveniles around $0.1 \mathrm{~g}$. At this size growth slows down, but individuals which become sufficiently large grow faster due to cannibalism, and reach their asymptotic size without encountering further bottlenecks. Cannibalism results in increased mortality and therefore a steeper size-spectrum. This state is referred to as the cannibalistic state. Compared to the resource driven state it has a higher impact on the resource, and consequently also larger total biomass. At high resource levels the elevated food abundance allows individuals to invade in small numbers and reach the cannibalistic state; this results in bistability being less widespread at high resource levels (Fig. 2).

The analysis of the two-species system is more complex but can be understood with reference to the single-species states. Coexistence states may be categorised based on whether the bottleneck in feeding level occur at or before size at maturation of the largest species as either competitive coexistence, corresponding to the resource-driven single species state (bottleneck at $m^{*}$ ), or trophic ladder coexistence, resembling the cannibalistic single species state (bottleneck in earlier life-stage). The two types of coexistence states are illustrated by two examples (Fig. 4). Competitive coexistence states are characterized by 1) an energetic bottleneck encountered just above the size of maturation of the larger species, 2) the two species have similar $m^{*}$ such that 
the $m^{*}$ ratio is smaller than the preferred predator-prey mass ratio $\left(m_{2}^{*} / m_{1}^{*}<\beta\right)$, and 3$)$ each of them are separately able to invade a pristine system comprised only of the resources (Fig. 2). Trophic ladder coexistence is characterised by 1) an energetic bottleneck in intermediate lifestages of the large species, 2) larger differences in $m^{*}$ between the two species $\left(m_{2}^{*} / m_{1}^{*}>\beta\right)$, and 3 ) the smaller species may be critical to allow the larger species to grow to size at maturation and hence sustain its population. The larger species may thus use the small species as a ladder to obtain a higher trophic position. It is noted that it is only large species above the dashed line in Fig. 2.a-b that cannot persist without the smaller species, while this gradually relaxes below the dashed line.

Whether two species can coexist depends on the resource level and the ratio between their sizes of maturation (Fig. 5). The most important findings in the coexistence analysis are: 1) Coexistence states above the dashed line (panels a and b), and to the right of the curved line (panels $\mathrm{c}$ and $\mathrm{d}$ ), are all trophic ladder states, where the largest species cannot invade from small numbers without the presence of the smaller species. 2) Below the dashed line trophic ladder states are found when the differences in $m^{*}$ between the two species is large; i.e. $m_{2}^{*} / m_{1}^{*}>\beta(\mathrm{a}, \mathrm{b})$, while competitive states are mainly found when the species have similar $m^{*}$; i.e. $\left(m_{2}^{*} / m_{1}^{*}<\beta\right)$ (a). It is not possible to draw distinct boundaries between the two coexistence states since the distinction between the two types of coexistence is only qualitative; a continuous transition between the states occur in phase space (a, c). 3) When resource level is increased the maximum allowed size for the largest species increases (a-c), and 4) species need to have more different $m^{*}$ to coexist $(\mathrm{b}, \mathrm{c})$. Trophic ladder states therefore dominate at high resource levels. 5) For all resource levels a little above the critical feeding level coexistence is possible provided that the smallest species is small enough (c vs d), and 6) trophic ladder states can be found for all resources levels above $\approx 0.26(\mathrm{c})$. When 7$)$ the size of the small species increases the competitive coexistence states disappear (c vs d). 8) For a specific pair of species $\left(m^{*}\right.$ of the two species are fixed) coexistence is only possible in a finite resource range (c). 9) When performing the coexistence analysis we did not encounter any alternative states where both species are present simultaneously, i.e. no alternative coexistence states were encountered.

As selection of prey in the model only depends on the relative prey size all species have the potential of being cannibals if they become large enough to eat their own offspring. However cannibalism and predation differ with cannibalism being an intraspecific process compared to predation that operates interspecifically. The importance of cannibalism is examined by introducing and systematically varying a fixed cannibalistic preference $\Theta_{c}$ for each species (M1); see Hartvig et al. (2011) for details. At low cannibalistic preferences coexistence states from Fig. 4 collapse to single species states (Fig. 6). However, a modest cannibalistic preference enables coexistence (preference larger than 0.35 in the case of Fig. 6). It is noted that even for full (neutral) preferences of 1 the actual diet due to cannibalism rarely exceeds 20-30\%, and that coexistence is possible over a large range of preferences where the diet from cannibalism is less than $10 \%$. Furthermore, cannibalism is often more pronounced in single-species states compared to coexistence states; this is due to a higher abundance of suitable prey from the smaller species than suitable conspecifics (cf. Fig. 4). A low content of conspecifics in the realised diet is thus a result of population size structure rather than a lacking capability of being a cannibal.

A community of more than two coexisting species may be assembled by sequential introduction of new species through invasion in small numbers (Fig. 7). Depending on the $m^{*}$ of the invader there are four outcomes of the invasion: 1) the invader may not invade, 2) the invader may invade and coexist with the resident(s), 3) the invader may expel the resident(s) (Fig. 7.D2), or 4) the invader causes a reconfiguration of the system, such that both the invader and a resident(s) are excluded (Fig. 7.C, light grey). The last possibility is an example of an invader-induced attractor shift which is described in Appendix B. After each additionally added species the scope for coexistence of yet another species shrinks (black bars in each panel). In 
the example provided there is potential for coexistence of four species (Fig. 7.D1). Coexistence of four species is, however, a rare occurrence, meaning that coexistence of two and in some cases three species is the most likely outcome of an assembly process (Fig. 8).

\section{Discussion}

\section{Single species states}

A single species with ontogenetic trophic niche shifts living on a resource is known to have the potential for several stable states (Claessen and de Roos, 2003; de Roos et al., 2003; van Kooten et al., 2005). These states are also found here and can be understood in terms of an energetic bottleneck. Through invasion in small numbers a resource-driven state may be reached where the population primarily feed on the resource spectrum. The maximum body size a species can obtain is determined by the energetic bottleneck individuals experience when they become too large to efficiently feed on the resources. The resource-driven state corresponds to the "stunted" state described in Claessen and de Roos (2003) where adult growth stops just after maturation, and thus before the maximum feasible body size, due to the energetic bottleneck (Claessen et al., 2002). The productivity of the resources determines the maximum sized $m^{*}$ species that can invade. Invasion in large numbers, or invasion in small numbers in resource-rich environments, allows a species to enter a high biomass cannibalistic state (Claessen and de Roos, 2003) where the extra food from conspecifics helps individuals through the energetic bottleneck. The cannibalistic state extends into areas of phase space where invasion into the resource driven state is not feasible leading to an emergent Allee effect (de Roos et al., 2003). In other areas of phase space where the cannibalistic state occurs the population may also be in the resource driven state. Hence there is scope for bistability as described by van Kooten et al. (2005). Our analysis demonstrates that both emergent Allee effects and bistability are widespread in phase space and can thus be expected to be commonplace for a cannibalistic single population with ontogenetic trophic niche shifts.

\section{Two species coexistence}

The flow of energy between species is an emergent result of the individual-level interactions, and it may vary from exploitative competition for resources, when the two species have similar and small adult sizes, to trophic chains and intraguild predation when one of the species has a larger adult size. We distinguish between two types of coexistence: 1) Competitive coexistence states where the species have similar sizes at maturation $\left(m_{2}^{*} / m_{1}^{*}<\beta\right)$ and they can both invade without the presence of the other. The size structures of the coexisting species are similar to the resource-driven single-species size structure. 2) Trophic ladder coexistence states where the ratio between size at maturation of the two species is larger than the preferred predator-prey mass ratio $\left(m_{2}^{*} / m_{1}^{*}>\beta\right)$, where the larger species needs the smaller species to obtain a higher trophic position. The combined size structure of the two species in the trophic ladder states is similar to single-species cannibalistic states, as predation on the smaller species allows the larger species to pass the energetic bottleneck.

Other studies have examined, and in some cases found, coexistence between two structured species populations. Hin et al. (2011) and Schellekens and van Kooten (2012) observed coexistence between two stage-structured intra-guild predators only when one of them acts as a predator and the other as a consumer. Thus the two species essentially divide the trophic niche space between them in a manner similar to the trophic ladder motif identified here. Another study concluded that size-dependent food intake and food-dependent growth, inherent in our size-structured model, demoted coexistence in an intraguild predation system where both species are size-structured (van de Wolfshaar et al., 2006). The model was parameterised for roach (Rutilus rutilus) and perch (Perca fluviatilis) and did not consider cannibalism. However, 
cannibalism in perch is common (Persson et al., 2000), and when included model studies show that cannibalism promotes coexistence between roach and perch (van de Wolfshaar, 2006). The model presented here is parameterised for fish in general to allow for a more general analysis of coexistence and is only capable of characterising roach and perch as two species with a relatively small ratio in sizes at maturation (maturation sizes of roach and perch is $14 \mathrm{~cm}$ and $16.8 \mathrm{~cm}$, and maximum published weights of $1.8 \mathrm{~kg}$ and $4.8 \mathrm{~kg}$, source: www.fishbase.org). As our results show that trophic ladder states are the most widespread coexistence states we may hypothesize that it is likely that van de Wolfshaar et al. (2006) would find coexistence along their resource gradient had they parametrised their model for roach and pikeperch (S. lucioperca) or perch and pike (Esox lucius).

In addition to the trophic ladder coexistence states we further identified the competitive coexistence states where the species are engaged in exploitative competition. Interestingly we find that competitive coexistence states are restricted to low to intermediate resource levels, where two species coexist on the range of resources provided by the resource spectrum. We find that no more than two resource driven species could coexist in the system. Interpreted in the light of limiting similarity theory (Hutchinson, 1959; MacArthur and Levins, 1967; Szabó and Meszéna, 2006) this means that the resource spectrum essentially can be partitioned into a maximum of two niches. When the resource level increases beyond the intermediate level only trophic ladder states are found, meaning that the resource spectrum only constitutes a single niche at high resource levels.

The trophic ladder coexistence state bears resemblance to an intraguild predation motif. In the trophic ladder states the smaller species is needed for the larger species to obtain a high trophic position. The reliance of the larger species on the smaller species is thus similar to a classic trophic chain motif (Oksanen et al., 1981; Hin et al., 2011) with the difference that the large species still competes with the smaller species as a juvenile. The state therefore bears closer resemblance to the intraguild predation motif with the additional effect of cannibalism (Polis et al., 1989; Diehl and Feißel, 2000; Rosenheim, 2007). It is noted that cannibalism only constitutes a smaller part of the diet (even for high cannibalistic preferences) as smaller conspecifics have a low abundance relative to similarly sized food items. That cannibalism is not required for driving this pattern is supported by Schellekens and van Kooten (2012) who find coexistence in an intra-guild system of stage-structured species that are not cannibalistic.

Classical theory based on unstructured population models predicts that intraguild predation coexistence only occur in a limited range of intermediate resource levels, as the consumer excludes the predator at low resource levels and vice versa at high levels (Holt and Polis, 1997; Mylius et al., 2001). This stands in contrast to empirical studies where coexistence is found at high resource levels (Amarasekare, 2008). Arim and Marquet (2004) examined the occurrence of intraguild predation in empirical food webs, and found it to be a widespread interaction in nature, pointing to a fundamental discrepancy between theory and data. In contrast to the competitive coexistence states we find the trophic ladder states to be present at all resource levels. Thus, we find that it indeed is possible to locate species pairs that can coexist in an IGP-like motif at all resource levels, but that each specific pair only coexists over a finite range of resource levels. This indicates that size-based feeding and the associated ontogenetic niche shifts may play an important role in mediating coexistence in purely size-structured intraguild predation-like motifs in e.g. aquatic communities.

The emergent Allee effects in the single-species states enables the possibility of the "resident strikes back"-phenomena in the two-species case (Mylius and Diekmann, 2001; Geritz et al., 2002): If the resident species is in a resource-driven state in an area of phase space with potential for bistability, the invader may drive the resident to change attractor to the cannibalistic state. Once in the cannibalistic state, the resident drives the invader to extinction. We have observed this phenomenon at intermediate, but not at high resource levels (Appendix B). 


\section{Cannibalism}

Cannibalism is known to be widespread among fish (Smith and Reay, 1991) as well as across taxa (Fox, 1975; Polis, 1981; Elgar and Crespi, 1992). In the size-structured model the potential for cannibalism is a consequence of the assumption that prey selection is only due to relative prey size and can thus occur whenever the ratio between the size at maturation and offspring size is larger than the preferred predator-prey mass ratio. Cannibalism is central to the cannibalistic single-species state where adults rely on food from conspecifics. Coexistence of two species may occur even if the cannibalistic preference is lowered but a modest preference towards cannibalism increase the scope for coexistence of two species. Even though cannibalism facilitates coexistence the adults of the larger species are not solely relying on cannibalism as their diet is dominated by the resource in competitive coexistence and by the smaller species in trophic ladder coexistence.

That cannibalism increases the scope for coexistence is in line with established knowledge showing that cannibalism can enhance intraguild predation coexistence in unstructured models (Rudolf, 2007; Amarasekare, 2008), which again relates to the general finding that coexistence requires the integrated sum of direct (e.g. cannibalism) and indirect (feedback loops from the focal species through both prey and predators) intraspecific interaction components to outweigh the total interspecific interaction component (Chesson and Kuang, 2008).

\section{Model architecture}

With the trait-based approach we suggest a way of performing more general studies of coexistence where the species identity can be varied through the trait-based species characterisation. This stands in contrast to most previous approaches that focuses on a parameter range (e.g. over a resource gradient) at which a given set of species can coexist.

The cost of generality is the loss of species-specific details; parameters (Table 2) do naturally vary across species, which may partly be accommodated by increasing trait dimensionality (cf. next section). We describe the types of states and their location in phase space for "mean species" (Hartvig et al., 2011), and changes to parameters such as $\beta$ and $\eta$ will change the location and area in phase space of the different state types: Changing preferred predator-prey mass ratio $\beta$ will shift trophic ladder states to occur for different $m^{*}$ ratios (cf. previous section). The parameter $\eta$ determines the size range the adult population covers where the most extreme variation is going from the mean value to the case of determinate growth $(\eta=1)$. However, even for this case both state types will persist as the bistability remains in stage-structured models having only a single juvenile and a single adult stage (van Kooten et al., 2005). We therefore expect that changes in model parameters will affect our results quantitatively but not qualitatively.

\section{Increasing species richness}

A natural next step beyond examining the coexistence of two species is to add a third species. The scope for coexistence of three species is much smaller than for two species. Coexistence of four species is rare but may be achieved; however we have been unable to find systems with coexistence of five species. This shows that large food webs of diverse size-structured species populations cannot be realised with a one-dimensional trait characterisation.

Generally speaking, coexistence requires a low niche overlap, which in turn is determined by the overlap of both prey and predators, or by a decreasing difference in relative fitness between species when their niche overlap increase (Chesson and Kuang, 2008). A decreased overlap in niche space may be obtained by increasing the dimensionality of the trait-based species characterisation as this allows for increased dimensionality of the niche space (Rossberg et al., 2010a,b). An increased niche space dimensionality increases the ways in which the overlap can be lowered. Thus, increasing trait dimensionality allows accounting for more of the details that make up specific species as it e.g. allows species to have similar prey, but different predators - 
or vice versa. It has indeed been demonstrated that species rich systems may be achieved for higher trait-dimensionality in both size-structured (Hartvig, 2011) and unstructured systems (Zhang et al., ress), and that once established the trait-diversity promotes dynamic stability in size-structured communities (Zhang et al., 2012), as the increased diet breath increases the number of weak links (McCann et al., 1998).

Obvious trait candidates for increased dimensionality include species-specific preferred predatorprey mass ratios as this allows transfer of energy from different trophic levels for predators of equal sizes. An alternative route to increased trait-dimensionality is to include a species-specific preference matrix as in Hartvig et al. (2011).

\section{Acknowledgements}

M.H. acknowledges support from European Marie Curie RTN FishACE through the EU's 6th FP (MRTN-CT-2004-005578) and the Danish National Research Foundation for support to the Center for Macroecology, Evolution and Climate. K.H.A. was supported by the Villum Kann Rasmussen Centre of Excellence: Ocean Life, and the EU's 7th framework research projects FACTS and MYFISH.

\section{References}

Amarasekare, P. (2008). Coexistence of intraguild predators and prey in resource-rich environments. Ecology, 89(10):2786-2797.

Andersen, K. H. and Beyer, J. E. (2006). Asymptotic size determines species abundance in the marine size spectrum. Am. Nat., 168(1):54-61.

Andersen, K. H., Beyer, J. E., Pedersen, M., Andersen, N. G., and Gislason, H. (2008). Life-history constraints on the success of the many small eggs reproductive strategy. Theor. Popul. Biol., 73(4):490497.

Arim, M. and Marquet, P. A. (2004). Intraguild predation: a widespread interaction related to species biology. Ecol. Lett., 7(7):557-564.

Barnes, C., Maxwell, D., Reuman, D. C., and Jennings, S. (2010). Global patterns in predator-prey size relationships reveal size dependency of trophic transfer efficiency. Ecology, 91(1):222-232.

Beverton, R. J. H. and Holt, S. J. (1957). On the dynamics of exploited fish populations. Fishery Investigations Series II, XIX. Ministry of Agriculture, Fisheries and Food.

Brose, U., Jonsson, T., Berlow, E. L., Warren, P., Banasek-Richter, C., Bersier, L.-F., Blanchard, J. L., Brey, T., Carpenter, S. R., Blandenier, M.-F. C., Cushing, L., Dawah, H. A., Dell, T., Edwards, F., Harper-Smith, S., Jacob, U., Ledger, M. E., Martinez, N. D., Memmott, J., Mintenbeck, K., Pinnegar, J. K., Rall, B. C., Rayner, T. S., Reuman, D. C., Ruess, L., Ulrich, W., Williams, R. J., Woodward, G., and Cohen, J. E. (2006). Consumer-resource body-size relationships in natural food webs. Ecology, 87(10):2411-2417.

Chesson, P. and Kuang, J. J. (2008). The interaction between predation and competition. Nature, 456(7219):235-238.

Claessen, D. and de Roos, A. M. (2003). Bistability in a size-structured population model of cannibalistic fish - a continuation study. Theor. Popul. Biol., 64(1):49-65.

Claessen, D., Van Oss, C., de Roos, A., and Persson, L. (2002). The impact of size-dependent predation on population dynamics and individual life history. Ecology, 83(6):1660-1675.

Cohen, J. E., Pimm, S. L., Yodzis, P., and Saldaña, J. (1993). Body sizes of animal predators and animal prey in food webs. J. Anim. Ecol., pages 67-78. 
de Roos, A. M. and Persson, L. (2001). Physiologically structured models - from versatile technique to ecological theory. Oikos, 94:51-71.

de Roos, A. M. and Persson, L. (2013). Population and community ecology of ontogenetic development, volume 51. Princeton University Press.

de Roos, A. M., Persson, L., and Thieme, H. R. (2003). Emergent Allee effects in top predators feeding on structured prey populations. Proc. R. Soc. Lond. B, 270:611-618.

de Roos, A. M., Schellekens, T., Kooten, T. V., Wolfshaar, K. V. D., Claessen, D., and Persson, L. (2008a). Simplifying a physiologically structured population model to a stage-structured biomass model. Theor. Popul. Biol., 73:47-62.

de Roos, A. M., Schellekens, T., Van Kooten, T., and Persson, L. (2008b). Stage-specific predator species help each other to persist while competing for a single prey. Proc. Natl. Acad. Sci. USA, 105(37):13930-13935.

Diehl, S. and Feißel, M. (2000). Effects of enrichment on three-level food chains with omnivory. Am. Nat., 155(2):200-218.

Drake, J. A. (1990). The Mechanics of Community Assembly and Succession. J. Theor. Biol., 147(2):213233.

Duarte, C. M. and Alcaraz, M. (1989). To produce many small or few large eggs: a size-independent reproductive tactic of fish. Oecologia, 80:401-404.

Elgar, M. and Crespi, B. (1992). Cannibalism: Ecology and Evolution among Diverse Taxa. Oxford University Press.

Fenchel, T. (1974). Intrinsic Rate of Natural Increase: The Relationship with Body Size. Oecologia, 14:317-326.

Fox, L. (1975). Cannibalism in natural populations. Annu. Rev. Ecol. Evol. Syst., 6:87-106.

Geritz, S. A. H., Gyllenberg, M., Jacobs, F. J. A., and Parvinen, K. (2002). Invasion dynamics and attractor inheritance. J. Math. Biol., 44:548-560.

Guill, C. (2009). Alternative dynamical states in stage-structured consumer populations. Theor. Popul. Biol., 76(3):168-178.

Hartvig, M. (2011). Ecological processes yield complex and realistic food webs. In Food Web Ecology individual life-histories and ecological processes shape complex communities, pages 75-99. Ph.D. Thesis, Lund University, Department of Biology. ISBN: 978-91-7473-080-7.

Hartvig, M., Andersen, K. H., and Beyer, J. E. (2011). Food web framework for size-structured populations. J. Theor. Biol., 272(1):113-122.

Hin, V., Schellekens, T., Persson, L., and de Roos, A. M. (2011). Coexistence of predator and prey in intraguild predation systems with ontogenetic niche shifts. The American Naturalist, 178(6):701-714.

Holt, R. D. and Polis, G. A. (1997). A theoretical framework for intraguild predation. Am. Nat., 149(4):745-764.

Hutchinson, G. E. (1959). Homage to Santa Rosalia or Why Are There So Many Kinds of Animals? Am. Nat., 93(870):145-159.

Law, R. (1999). Theoretical aspects of community assembly. In McGlade, J., editor, Advanced Ecological Theory - Principles and Applications, chapter 5, pages 143-171. Blackwell Science.

MacArthur, R. and Levins, R. (1967). The limiting similarity, convergence, and divergence of coexisting species. Am. Nat., 101(921):377-385. 
McCann, K., Hastings, A., and Huxel, G. R. (1998). Weak trophic interactions and the balance of nature. Nature, 395:794-798.

Metz, J. A. J. and Diekmann, O., editors (1986). The Dynamics of Physiologically Structured Populations, volume 68 of Lecture Notes in Biomathematics. Springer-Verlag.

M'Kendrick, A. G. (1926). Applications of Mathematics to Medical Problems. P. Edinburgh Math. Soc., 44:98-130.

Mylius, S. D. and Diekmann, O. (2001). The Resident Strikes Back: Invader-Induced Switching of Resident Attractor. J. Theor. Biol., 211:297-311.

Mylius, S. D., Klumpers, K., de Roos, A. M., and Persson, L. (2001). Impact of intraguild predation and stage structure on simple communities along a productivity gradient. Am. Nat., 158(3):259-276.

Oksanen, L., Fretwell, S., Arruda, J., and Niemela, P. (1981). Exploitation ecosystems in gradients of primary productivity. Am. Nat., 118(2):240.

Persson, L., Byström, P., and Wahlström, E. (2000). Cannibalism and competition in eurasian perch: population dynamics of an ontogenetic omnivore. Ecology, 81(4):1058-1071.

Peters, R. H. (1983). The ecological implications of body size. Cambridge University Press.

Pimm, S. L. and Rice, J. C. (1987). The dynamics of multispecies, multi-life-stage models of aquatic food webs. Theor. Popul. Biol., 32(3):303-325.

Polis, G. A. (1981). The evolution and dynamics of intraspecific predation. Annu. Rev. Ecol. Evol. Syst., 12:225-251.

Polis, G. A., Myers, C. A., and Holt, R. D. (1989). The ecology and evolution of intraguild predation: potential competitors that eat each other. Annu. Rev. Ecol. Evol. Syst., 20:297-330.

Post, W. M. and Pimm, S. L. (1983). Community assembly and food web stability. Math. Biosci., 64(2):169-182.

Press, W. H., Flannery, B. P., Teukolsky, S. A., and Vetterling, W. T. (1992). Numerical Recipes in $C$ : The Art of Scientific Computing. Cambridge University Press. Also available at http://www.nr.com/.

Rosenheim, J. A. (2007). Intraguild Predation: New Theoretical and Empirical Perspectives. Ecology, 88 (Special Feature):2679-2680.

Rossberg, A. G., Brännström, A., and Dieckmann, U. (2010a). Food-web structure in low-and highdimensional trophic niche spaces. J. R. Soc. Interface.

Rossberg, A. G., Brännström, Å., and Dieckmann, U. (2010b). How trophic interaction strength depends on traits. Theor. Ecol., 3(1):13-24.

Rudolf, V. (2007). The interaction of cannibalism and omnivory: consequences for community dynamics. Ecology, 88(11):2697-2705.

Savage, V. M., Gillooly, J. F., Brown, J. H., West, G. B., and Charnov, E. L. (2004). Effects of Body Size and Temperature on Population Growth. Am. Nat., 163(3):429-441.

Schellekens, T. and van Kooten, T. (2012). Coexistence of two stage-structured intraguild predators. J. Theor. Biol., 308(0):36 - 44 .

Smith, C. and Reay, P. (1991). Cannibalism in teleost fish. Rev. Fish Biol. Fisher., 1(1):41-64.

Szabó, P. and Meszéna, G. (2006). Limiting similarity revisited. Oikos, 112:612-619.

Ursin, E. (1973). On the Prey Size Preferences of Cod and Dab. Meddelelser fra Danmarks Fiskeri- og Havundersøgelser, N.S, 7:85-98. 
Ursin, E. (1974). Search Rate and Food Size Preference in Two Copepods. ICES CM, L/23:1-13.

van de Wolfshaar, K. E. (2006). Chapter 6: Interspecific competitive bottlenecks in intraguild predation systems - model predictions and field data. In Population persistence in the face of size-dependent predation and competition interactions, pages 97-122. Ph.D. thesis, University of Amsterdam, The Netherlands.

van de Wolfshaar, K. E., de Roos, A. M., and Persson, L. (2006). Size-Dependent Interactions Inhibit Coexistence in Intraguild Predation Systems with Life-History Omnivory. Am. Nat., 168(1):62-75.

van Kooten, T., de Roos, A. M., and Persson, L. (2005). Bistability and an Allee effect as emergent consequences of stage-specific predation. J. Theor. Biol., 237:67-74.

von Foerster, H. (1959). Some Remarks on Changing Populations. In Stohlman, F., editor, The Kinetics of Cellular Proliferation, pages 382-407. Grune \& Stratton.

Warren, P. H. and Lawton, J. H. (1987). Invertebrate predator-prey body size relationships: an explanation for upper triangular food webs and patterns in food web structure? Oecologia, 74(2):231-235.

Werner, E. E. and Gilliam, J. F. (1984). The Ontogenetic Niche and Species Interactions in SizeStructured Populations. Annu. Rev. Ecol. Evol. Syst., 15:393-425.

Winemiller, K. O. and Rose, K. A. (1993). Why do most fish produce so many tiny offspring? Am. Nat., 142(4):585-603.

Zhang, L., Hartvig, M., Knudsen, K., and Andersen, K. H. (In press). Size-based predictions of food web patterns. Theoretical Ecology.

Zhang, L., Thygesen, U., Knudsen, K., and Andersen, K. (2012). Trait diversity promotes stability of community dynamics. Theor. Ecol., pages 1-13. 10.1007/s12080-012-0160-6.

Supplementary online material: Appendix A-B and Fig. S1-S5. 
a) Interactions of larvae

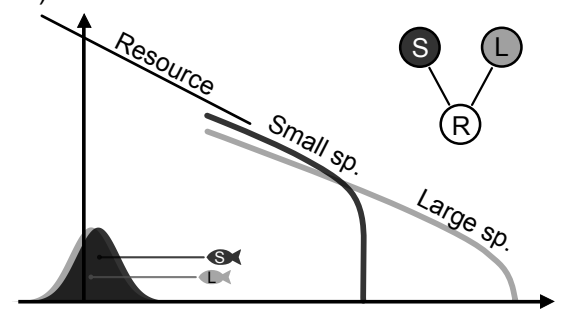

b) Interactions of juveniles
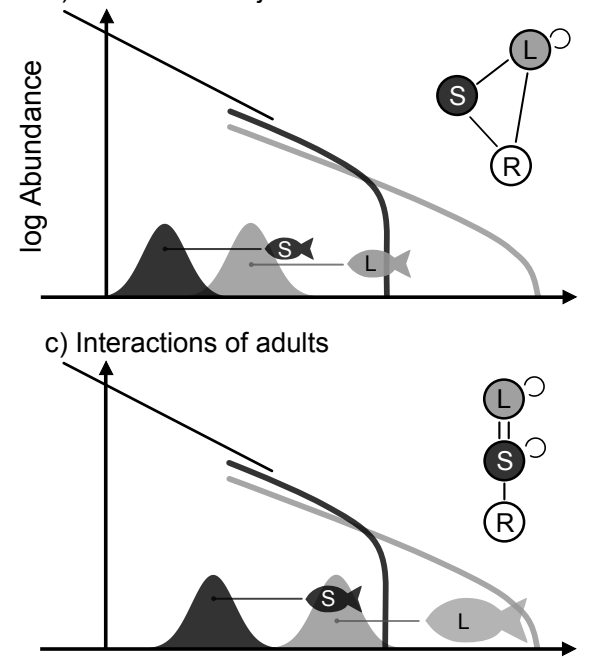

$\log$ Mass

Figure 1: Illustration of how different interactions between individuals emerge depending on body size from two populations of species with a small (black) and large size at maturation (grey); solid lines shows the abundance distributions of the two species along with a continuous resource spectrum (thin black line). Each panel focuses on individuals with different sizes (fish pictograms) and shows their food preference with a grey or black bell-shaped patch along with their interaction motif in the upper right corner. a) Two larvae or small juveniles of the same size compete for food from the resource spectrum and interact in an exploitative competition motif. b) Juveniles interact in an intraguild-predation type of interaction where the larger juveniles from the large species have a diet consisting of the resource and the smaller species along with some cannibalism. c) Adults from both populations can prey on the competing population, but the link from the small to the large species is weaker, such that the effective motif resembles a trophic chain with cannibalism. At the species level the resulting interaction motif will be a mix of these individual-level motifs, and the species-level motif depends on both the differences in size at maturation as well as the specific size-structures of the populations. The specific shapes of the size distributions are drawn using equilibrium theory where growth and mortality are kept constant (Andersen and Beyer, 2006; Hartvig et al., 2011). 
Table 1: Model equations. $m$ is individual mass, $m_{p}$ is mass of a prey item, and $m^{*}$ is the species-specific trait size at maturation.

\begin{tabular}{|c|c|c|c|}
\hline Community size-spectrum & $N_{c}(m)=N_{R}(m)+\sum_{i} N_{i}(m)$ & $\# /\left(\mathrm{g} \mathrm{m}^{3}\right)$ & $(\mathrm{M} 1)$ \\
\hline Resource dynamics & $\frac{\partial N_{R}(m)}{\partial t}=r_{0} m^{p-1}\left[\kappa m^{-\lambda}-N_{R}(m)\right]-\mu_{p}(m) N_{R}(m)$ & $\# /\left(\mathrm{yrg} \mathrm{m}^{3}\right)$ & $(\mathrm{M} 2)$ \\
\hline Volumetric search rate & $v(m)=\gamma m^{q}$ & $\mathrm{~m}^{3} / \mathrm{yr}$ & (M3) \\
\hline Feeding kernel & $s\left(m_{p} / m\right)=\exp \left[-\ln ^{2}\left(\beta m_{p} / m\right) /\left(2 \sigma^{2}\right)\right]$ & {$[0 ; 1]$} & $(\mathrm{M} 4)$ \\
\hline Encountered food & $\varepsilon(m)=v(m) \int s\left(m_{p} / m\right) N_{c}\left(m_{p}\right) m_{p} d m_{p}$ & $\mathrm{~g} / \mathrm{yr}$ & $(\mathrm{M} 5)$ \\
\hline Feeding level & $f(m)=\varepsilon(m) /\left[\varepsilon(m)+h m^{n}\right]$ & {$[0 ; 1]$} & $(\mathrm{M} 6)$ \\
\hline Available energy & $E(m)=\alpha f(m) h m^{n}-k m^{p}$ & $\mathrm{~g} / \mathrm{yr}$ & $(\mathrm{M} 7)$ \\
\hline Allocation to reproduction & $\psi\left(m, m^{*}\right)=\left[1+\left(\frac{m}{m^{*}}\right)^{-u}\right]^{-1}\left(\frac{\eta m}{m^{*}}\right)^{1-n} \quad$ for $n=p^{\dagger}$ & {$[0 ; 1]$} & $(\mathrm{M} 8)$ \\
\hline Somatic growth & $g\left(m, m^{*}\right)= \begin{cases}{\left[1-\psi\left(m, m^{*}\right)\right] E(m)} & E(m)>0 \\
0 & \text { otherwise }\end{cases}$ & $\mathrm{g} / \mathrm{yr}$ & (M9) \\
\hline Reproduction & $R_{i}=\frac{\epsilon}{2 m_{0}} \int N_{i}(m) \psi\left(m, m^{*}\right) \max \{E(m), 0\} d m$ & $\# /\left(\mathrm{yr} \mathrm{m}^{3}\right)$ & $(\mathrm{M} 10)$ \\
\hline Predation mortality & $\mu_{p}\left(m_{p}\right)=\int s\left(m_{p} / m\right)[1-f(m)] v(m) N_{c}(m) d m$ & $\mathrm{yr}^{-1}$ & $(\mathrm{M} 11)$ \\
\hline Starvation mortality & $\mu_{s}(m)= \begin{cases}0 & E(m)>0 \\
-E(m) /(\xi m) & \text { otherwise }\end{cases}$ & $\mathrm{yr}^{-1}$ & $(\mathrm{M} 12)$ \\
\hline Background mortality & $\mu_{b}\left(m^{*}\right)=\mu_{0} m^{* n-1}$ & $\mathrm{yr}^{-1}$ & $(\mathrm{M} 13)$ \\
\hline
\end{tabular}

${ }^{\dagger}$ see Hartvig et al. (2011) for the equation governing the general case of $n \neq p$.

Table 2: Species-independent parameter values for a temperature of $10^{\circ} \mathrm{C}$ (Hartvig et al., 2011). Time is expressed in units of years (yr).

\begin{tabular}{|c|c|c|c|}
\hline \multicolumn{4}{|c|}{ Individual growth } \\
\hline$f_{0}$ & varied & - & Initial feeding level \\
\hline$\alpha$ & 0.6 & - & Assimilation efficiency \\
\hline$h$ & 85 & $\mathrm{~g}^{1-n} / \mathrm{yr}$ & Max. food intake \\
\hline$n$ & 0.75 & - & Exponent for max. food intake \\
\hline$k$ & 10 & $\mathrm{~g}^{1-p} / \mathrm{yr}$ & Std. metabolism and activity \\
\hline$p$ & 0.75 & - & Exponent of std. metabolism \\
\hline$\beta$ & 100 & - & Preferred pred.-prey mass ratio \\
\hline$\sigma$ & 1 & - & Width of feeding kernel \\
\hline$q$ & 0.8 & - & Exponent for search volume \\
\hline \multicolumn{4}{|c|}{ Reproduction } \\
\hline$m_{0}$ & 0.5 & $\mathrm{mg}$ & Offspring mass \\
\hline$\eta$ & 0.25 & - & $m^{*}$ rel. to asymptotic mass $M$ \\
\hline$\epsilon$ & 0.1 & - & Efficiency of offspring production \\
\hline$u$ & 10 & - & Width of maturation transition \\
\hline \multicolumn{4}{|c|}{ Mortality } \\
\hline$\xi$ & 0.1 & - & Fraction of energy reserves \\
\hline$\mu_{0}$ & 0.84 & $\mathrm{~g}^{1-n} / \mathrm{yr}$ & Background mortality \\
\hline \multicolumn{4}{|c|}{ Resource spectrum } \\
\hline$\kappa$ & $5 \cdot 10^{-}$ & $\mathrm{g}^{\lambda-1} / \mathrm{m}^{3}$ & Magnitude of resource spectrum \\
\hline$r_{0}$ & 4 & $\mathrm{~g}^{1-p} / \mathrm{yr}$ & Regeneration rate of resources \\
\hline$m_{c}$ & 0.5 & $\mathrm{~g}$ & Upper limit of resource spectrum \\
\hline \multicolumn{4}{|c|}{ Derived parameters } \\
\hline \multicolumn{3}{|c|}{$\gamma\left(f_{0}\right)=\frac{f_{0} h \beta^{2-\lambda}}{\left(1-f_{0}\right) \sqrt{2 \pi} \kappa \sigma}$} & Search volume $\left(\mathrm{m}^{3} \mathrm{~g}^{-q} / \mathrm{yr}\right)$ \\
\hline \multicolumn{3}{|c|}{$\lambda=2-n+q=2.05$} & Exponent of resource spectrum \\
\hline
\end{tabular}




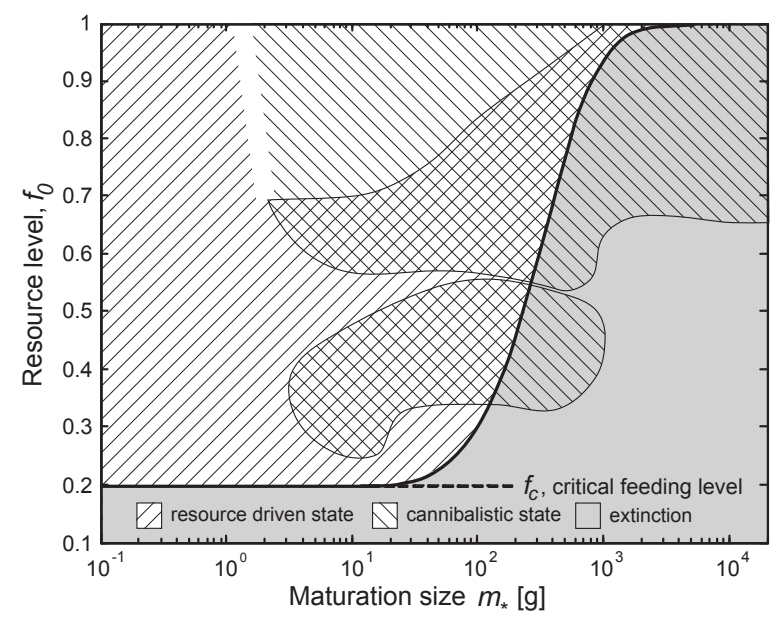

Figure 2: Single-species population states as a function of maturation size and resource level. Invasion from small numbers is possible if the resource level is above the critical level and if the species' maturation size is small enough to allow survival on resources only (white background). Hatching type indicates if the species has a resource-driven or a cannibalistic state (cf. legend). Areas with cross-hatching indicates bistability between the two states, i.e. the species may be in either the resource-driven or the cannibalistic state. The hatched grey area indicates an emergent Allee effect, where only the cannibalistic state exists. The white area in the top left quadrant marks a region where a continuous transition between states occurs.

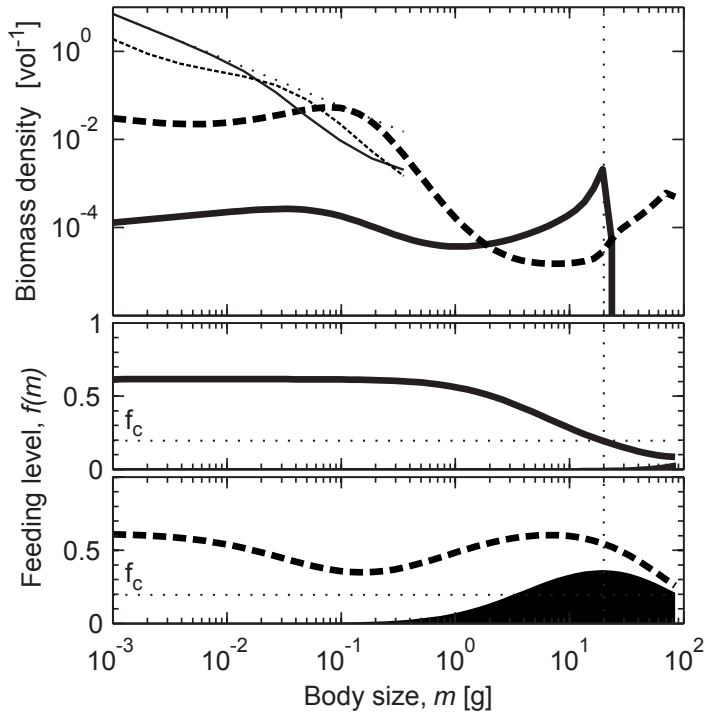

Figure 3: The two alternative single-species states: resource-driven (full) and cannibalistic (dashed) for $f_{0}=0.6$ and $m^{*}=20 \mathrm{~g}$. Upper panel: Biomass spectrum of species (thick lines) and resources (thin lines) along with carrying capacity of resources (dotted). Lower panels: Feeding level (thick line), feeding level resulting from cannibalism (shaded), and critical feeding level (horizontal dots). Vertical dotted line indicates size at maturation. Note the bottlenecks where the feeding level approaches the critical feeding level around the size at maturation in the resource-driven state and around $0.1 \mathrm{~g}$ in the cannibalistic state. 


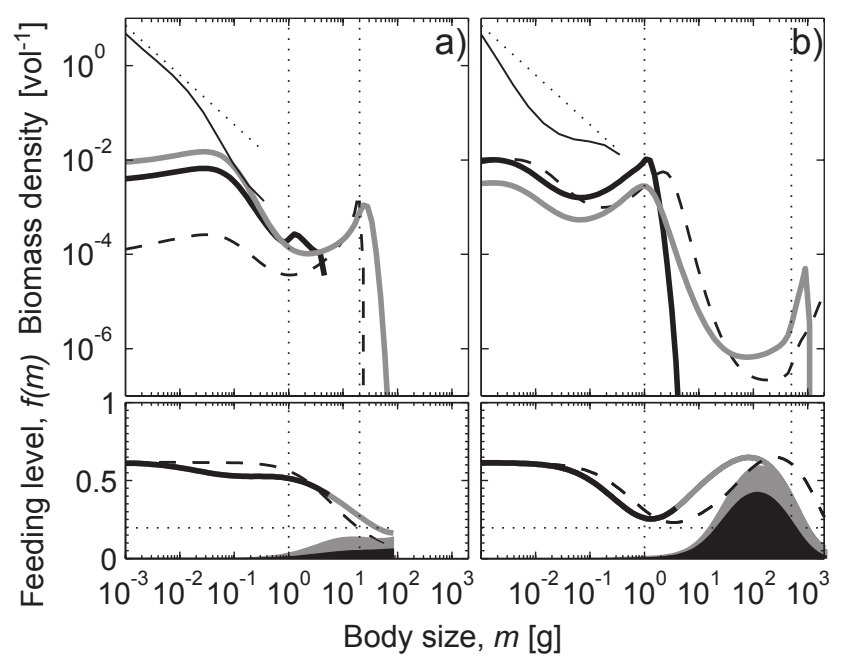

Figure 4: Illustration of the two types of coexistence states (solid lines) and their resemblance to single-species states of the larger species (dashed). a) Competitive coexistence for $f_{0}=0.6, m_{1}^{*}=1 \mathrm{~g}$ (black solid), and $m_{2}^{*}=20$ (grey solid) g, and the single-species resource-driven state for $m_{2}^{*}$ (black dashed, also shown in Fig. 3). b) Trophic ladder coexistence for $f_{0}=0.6, m_{1}^{*}=1 \mathrm{~g}$ (black solid), and $m_{2}^{*}=500 \mathrm{~g}$ (grey solid), and the singlespecies cannibalistic state for $m_{2}^{*}$ (black dashed). Upper panels: Biomass spectrum of species (thick lines) and resources (thin line) along with carrying capacity of resources (dotted). Lower panels: Feeding level for both species (overlapping black and grey solid lines), critical feeding level (horizontal dots), feeding level (for both species) resulting from feeding on the small species (black shading), the large species (grey shading), and the resources (white area below the curve). Also shown is the feeding level for the single-species state (black dashed). Vertical dotted lines indicate size at maturation. 

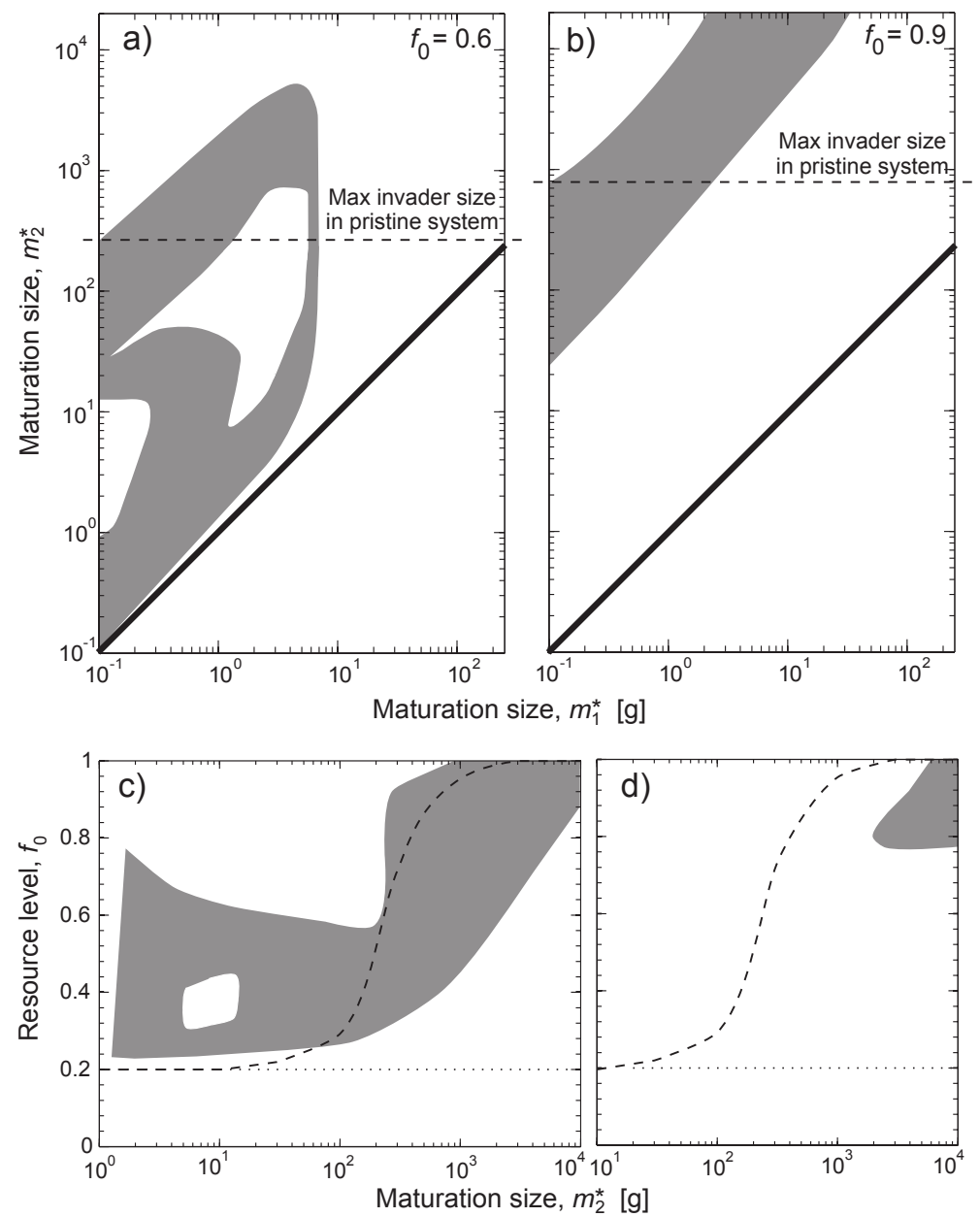

Figure 5: Coexistence (grey) of two species as a function of size at maturation for medium resource levels $\left(f_{0}=0.6\right.$; a) and high resource levels $\left(f_{0}=0.9 ; \mathrm{b}\right)$, and as a function of resource level $f_{0}$ for $m_{1}^{*}=1 \mathrm{~g}(\mathrm{c})$ and $m_{1}^{*}=10 \mathrm{~g}(\mathrm{~d})$. In white areas only one of the species can persist. For the given resource levels the dashed lines indicate the largest $m^{*}$ species that, in small numbers, can invade into a system comprised solely by the resource spectrum (a to d). Dotted lines show the critical feeding level $(\mathrm{c}, \mathrm{d})$. Coexistence states are mirrored around the diagonal line $(y=x)$, but only states above the line is shown to simplify visualisation $(a, b)$. States close to the diagonal are competitive coexistence states (a), whereas states further away are trophic ladder states (a, b); a gradual transition between the two types of states are present for intermediate resource levels $(\mathrm{a}, \mathrm{c})$. For low to intermediate resource level and small $m_{1}^{*}$ both competitive and trophic ladder states are found (a, c), whereas only trophic ladder states are found for high resource levels or an increased size of $m_{1}^{*}(b, d)$. 


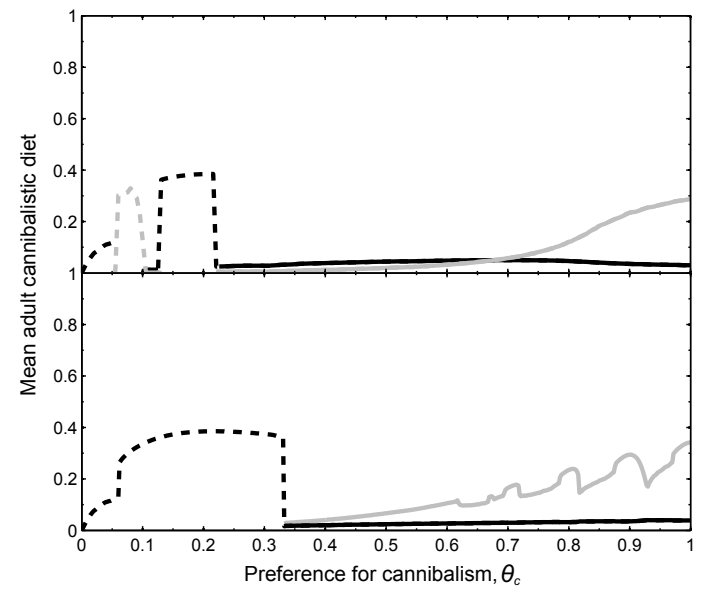

Figure 6: Fraction of adult diet from cannibalism as a function of the preference for cannibalism. The coexistence states from Fig. 4 collapse to single species states at low cannibalistic preferences. Top panel shows the cannibalistic diet in the competitive coexistence state from Fig. 4.a, and the lower panel shows the cannibalistic diet contribution in the trophic ladder state from Fig. 4.b. The sizes at maturation of the large (grey) and small (black) species are the same as in Fig. 4. Ranges of coexistence are marked with solid line, and single-species states with dashed lines. Oscillations for high preferences in the lower panel are due to amplitude changes in the population oscillations of the larger species.

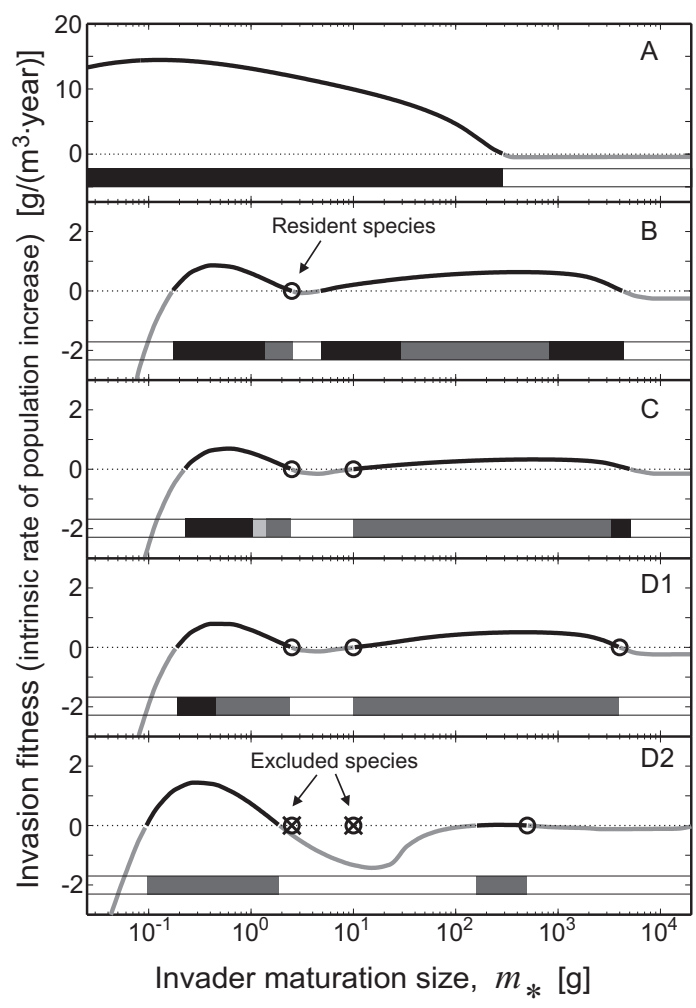

Figure 7: Assembly of a community from a pristine system and up to three coexisting species. Invasion fitness (rate of population increase when the invader is at low density) landscape of pristine system (A), one resident of $m_{1}^{*}=2.5 \mathrm{~g}(\mathrm{~B})$, two residents; $m_{1}^{*}$ and $m_{2}^{*}=10 \mathrm{~g}(\mathrm{C})$, three residents; $m_{1}^{*}, m_{2}^{*}$ and $m_{3}^{*}=4 \mathrm{~kg}$ (D1). (D2) illustrates the collapse of the community in (C) due to the introduction of an invader with $m^{*}=500 \mathrm{~g}$. Invasion fitness of the invader is shown with solid lines, which are black for positive values and grey for negative values. Under each landscape it is denoted whether invasion leads to coexistence (black), exclusion of one of the resident species (dark grey) or exclusion of both one of the resident species and the invader (light grey). 

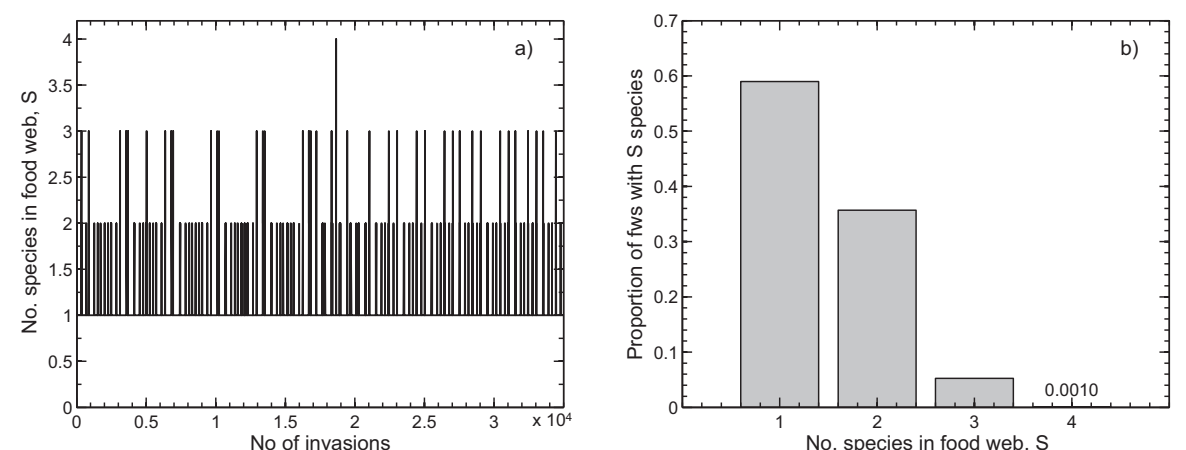

Figure 8: Sequential assembly of communities where species (size at maturation, $m^{*}$ ) are drawn randomly (uniform distribution of $\log \left(m^{*}\right)$ ). a) Number of coexisting species in the community during 35,000 invasion attempts. b) Distribution of the number of coexisting species during the assembly process. 


\section{Appendix A-B and Fig. S1-S5}

Online supplementary for Hartvig, M. and Andersen, K..H. 2013. Coexistence of structured populations with size-based prey selection. Theoretical Population Biology. doi:10.1016/j.tpb.2013.07.003.

\section{Appendix A}

\section{Numerical continuation}

To find the equilibria of the time-averaged model we exploit the property of structured populations that a state can be uniquely described by the reproduction of the population $R$ (Diekmann et al., 2003). We fix the value of the reproduction used in the boundary condition: $g\left(m_{0}\right) N\left(m_{0}\right)=R_{f i x}$, and simulate the solution forward till the resulting attractor is reached, where we calculate the actual reproduction $R$ using (M10) and average over time. At the time-averaged (as the attractor may be cyclic) equilibrium the reproductive value calculated using (M10) equals $R_{f i x}$. By varying the value of $R_{f i x}$ and recording the corresponding $R$ values a map can be constructed showing both stable and unstable fixed points (Fig. S1). By repeating this procedure for different values of a bifurcation parameter $\left(m^{*}\right.$ or $\left.f_{0}\right)$ a complete bifurcation diagram can be constructed (Fig. S2). This method is exact if the solution is constant in time, but only approximate if the solution is oscillatory. To find the exact solution in an oscillatory solution the predicted equilibrium is simulated forward in time to find the true solution.

To examine the coexistence of two species the method is generalized to varying the reproductive value of both species independently. Once a stable solution is found we vary a parameter, e.g. $m^{*}$, and search for the stable and unstable equilibria in the vicinity of the solution for the unvaried parameter. This search is done automatically and verified visually. The tricky part is naturally how much the parameter value should be varied to enable an effective search of the new equilibrium, and this is the problem more sophisticated methods solve. However, the outlined method proved easy and robust producing the diagrams in the paper (Fig. 2 and 5), which are made from calculations on a $25 \times 25$ point grid.

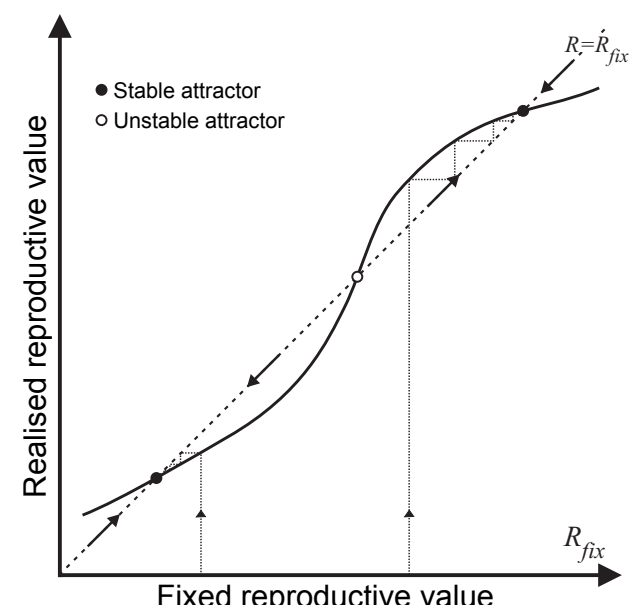

Fixed reproductive value
Fig. S1: Equilibria occurs when $R=R_{f i x}$. When $R$ is above $R_{f i x}$ a full model simulation will move the species towards a higher reproductive value, and oppositely when $R$ is below $R_{f i x}$. This allows determining whether an equilibrium is stable or unstable. This diagram represent a vertical transect of the situation at $f_{0}=0.6$ in Fig. S2. 


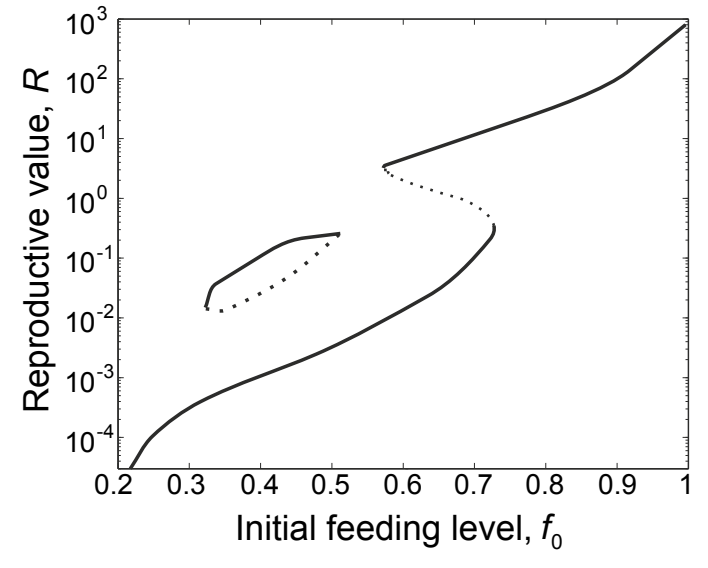

Fig. S2: Example of bifurcation diagram for $m^{*}=20 \mathrm{~g}$. This diagram constitutes a vertical transect of Fig. 2. Solid lines are stable equilibria (attractors), and dotted lines are unstable equilibria (repellers); meaning that if a species is introduced above a dotted line population dynamics will lead the species population to the equilibria above, and vice versa below.

\section{Appendix B}

\section{Community Assembly: Invader induced attractor shift (IIAS)}

When examining the assembly process (Fig. 7.C) one notices a peculiar scenario: the invader can initially invade (positive fitness landscape), but this invasion causes a reconfiguration of the community resulting in exclusion of one resident and the invader (Fig. S3). A similar possibility is the invasion of a species on a single resident species, where the invader initially has a positive invasion fitness, but is eventually not able to establish itself (Fig. S4). This phenomena is known as 'resident strikes back', or 'invader induced attractor shift' (Mylius and Diekmann, 2001; Geritz et al., 2002). What happens is that the biomass from the invader allows the resident(s) to change attractor to reach another stable configuration through changing its size-spectrum composition. Thus: invasion does not necessarily imply fixation, meaning that one have to turn to simulations to be sure of the outcome of a potentially feasible invasion.

The two species coexistence diagram (Fig. 5), may be enhanced to include the outcome in the cases where coexistence is not possible. Such a diagram (Fig. S5) resembles pairwise invasibility plots (PIPs) used in evolutionary ecology (van Tienderen and de Jong, 1986). We may term these plots pairwise invasion diagrams (PIDs), as they show the outcome of the invasion in contrast to the PIPs that show whether the invader can initially invade. Obtained PIDs (Fig. S5) show that regions where invader induced attractor shifts occur are not widespread, and can thus be considered an exotic phenomena.

PIDs are similar to PIPs, and may thus be used to predict the direction of evolution. In the case of medium resource level (Fig. S5.a) a single species will evolve towards smaller size at maturation $m^{*}$, as mutant (invader having a $m^{*}$ close to the resident) invasions will replace the resident when the mutant's $m^{*}$ is smaller than the resident's $m^{*}$. In resource-rich

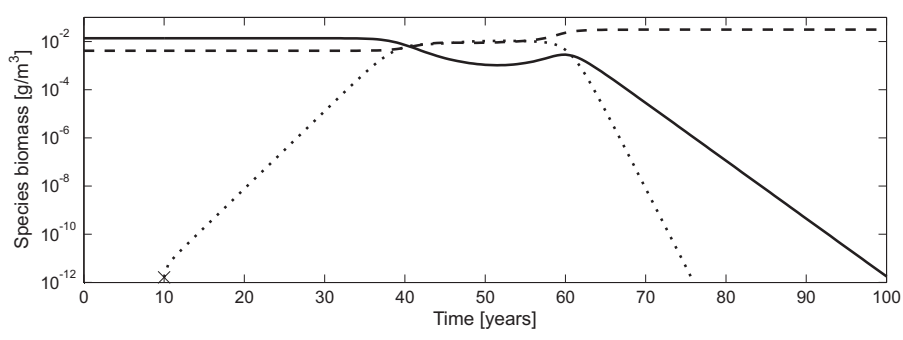

Fig. S3: Example of invader induced attractor shift (IIAS). Time evolution of the biomass of two residents $\left(m_{1}^{*}=2.5 \mathrm{~g}\right.$ solid, and $m_{2}^{*}=$ $10 \mathrm{~g}$ dashed lines), where an invader $\left(m_{I}^{*}=1.5 \mathrm{~g}\right.$, dotted line $)$ invades and causes a reconfiguration of the community. 

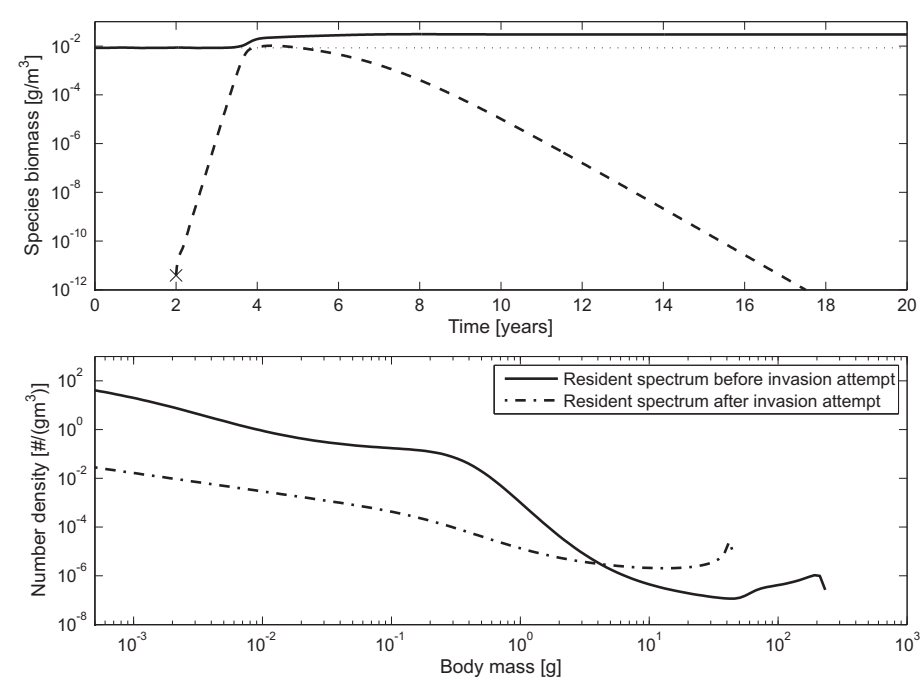

Fig. S4: Example of IIAS or 'resident strikes back' with one resident species $\left(m^{*}=55 \mathrm{~g}\right)$. Upper plot: time evolution of biomass for resident (solid line) and invader $\left(m^{*}=5 \mathrm{~g}\right.$, dashed line). Lower plot: size-spectrum of the resident before (solid line), and after the invasion attempt (dash-dotted line).

environments (Fig. S5.b) small residents will evolve towards larger $m^{*}$, and large residents will evolve towards smaller $\mathrm{m}^{*}$; at a size around $50 \mathrm{~g}$ the species reaches a singular point, where evolution can either come to a halt or cause speciation (Geritz et al., 1998).

\section{References}

Diekmann, O., Gyllenberg, M., and Metz, J. (2003). Steady-state analysis of structured population models. Theor. Popul. Biol., 63(4):309-338.

Geritz, S. A. H., Gyllenberg, M., Jacobs, F. J. A., and Parvinen, K. (2002). Invasion dynamics and attractor inheritance. J. Math. Biol., 44:548-560.

Geritz, S. A. H., Kisdi, E., Meszena, G., and Metz, J. A. J. (1998). Evolutionarily singular strategies and the adaptive growth and branching of the evolutionary tree. Evol. Ecol., 12:35-57.

Mylius, S. D. and Diekmann, O. (2001). The Resident Strikes Back: Invader-Induced Switching of Resident Attractor. J. Theor. Biol., 211:297-311.

van Tienderen, P. and de Jong, G. (1986). Sex ratio under the haystack model: Polymorphism may occur. J. Theor. Biol., 122(1):69 - 81.

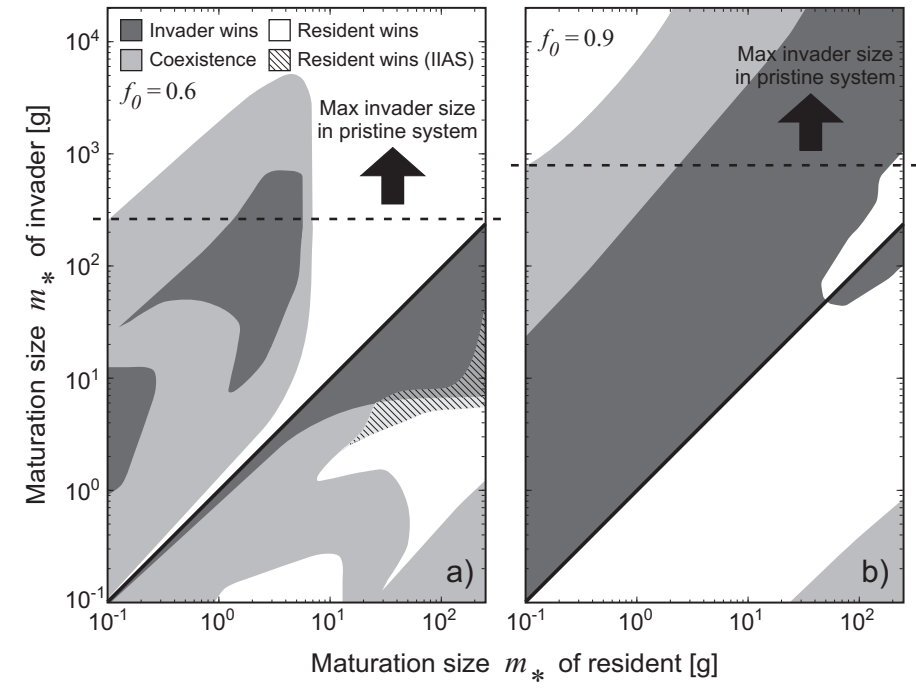

Fig. S5: Pairwise invasion diagrams (PIDs) showing the outcome of an invasion into a system having one resident species. a) PID for medium resource level $\left(f_{0}=0.6\right)$, and $\left.\mathrm{b}\right)$ high resource level $\left(f_{0}=0.9\right)$. PIDs show regions of co-existence (light grey), where invader excludes the resident (dark grey), where invasion is impossible (white), and where IIAS are present (hatched). The dashed line shows the maximum invader size that can invade a pristine community. 